\title{
Universal entanglement for higher dimensional cones
}

\author{
Pablo Bueno $^{a}$ and Robert C. Myers ${ }^{b}$ \\ ${ }^{a}$ Instituto de Física Teórica UAM/CSIC, \\ C/ Nicolás Cabrera, 13-15, C.U. Cantoblanco, Madrid, 28049 Spain \\ ${ }^{b}$ Perimeter Institute for Theoretical Physics, \\ 31 Caroline Street North, ON, N2L 2 Y 5 Canada \\ E-mail: p.bueno@csic.es, rmyers@perimeterinstitute.ca
}

AbStract: The entanglement entropy of a generic $d$-dimensional conformal field theory receives a regulator independent contribution when the entangling surface contains a (hyper)conical singularity of opening angle $\Omega$, codified in a function $a^{(d)}(\Omega)$. In arXiv:1505.04804, we proposed that for three-dimensional conformal field theories, the coefficient $\sigma^{(3)}$ characterizing the limit where the surface becomes smooth is proportional to the central charge $C_{\mathrm{T}}$ appearing in the two-point function of the stress tensor. In this paper, we prove this relation for general three-dimensional holographic theories, and extend the result to general dimensions. In particular, we define a generalized coefficient $\sigma^{(d)}$ to characterize the almost smooth limit of a (hyper)conical singularity in entangling surfaces in higher dimensions. We show then that this coefficient is universally related to $C_{\mathrm{T}}$ for general holographic theories and provide a general formula for the ratio $\sigma^{(d)} / C_{\mathrm{T}}$ in arbitrary dimensions. We conjecture that the latter ratio is universal for general CFTs. Further, based on our recent results in arXiv:1507.06997, we propose an extension of this relation to general Rényi entropies, which we show passes several consistency checks in $d=4$ and 6 .

KEYWORDS: Gauge-gravity correspondence, AdS-CFT Correspondence

ARXIV EPRINT: 1508.00587 


\section{Contents}

1 Introduction 1

2 Cone coefficients for $\mathrm{EE}$ in general dimensions 4

2.1 Three dimensions 6

2.2 General higher-dimensional case 8

3 Cone coefficients for Rényi entropy 11

$\begin{array}{lll}3.1 & \text { Four dimensions } & 12\end{array}$

$\begin{array}{lll}3.2 & \text { Six dimensions } & 15\end{array}$

$\begin{array}{lll}4 & \text { Discussion } & 18\end{array}$

$\begin{array}{ll}\text { A Twist operators } & 19\end{array}$

\section{Introduction}

Entanglement entropy (EE) and more generally Rényi entropy has long been seen as an interesting probe of quantum field theories (QFTs), e.g., [1-5]. Typically in this context, one chooses some region $V$ on a Cauchy surface (e.g., a constant time slice) and then evaluates the reduced density matrix $\rho_{V}$ by integrating out the degrees of freedom in the complementary region $\bar{V}$. The Rényi and entanglement entropies are then defined as

$$
S_{n}(V)=\frac{1}{1-n} \log \operatorname{Tr} \rho_{V}^{n}, \quad S_{\mathrm{EE}}(V)=\lim _{n \rightarrow 1} S_{n}(V)=-\operatorname{Tr}\left(\rho_{V} \log \rho_{V}\right) .
$$

The calculation of these quantities must be regulated, e.g., by a short distance cut-off $\delta$, because of an infinite number of short distance correlations in the vicinity of the entangling surface, i.e., the boundary of $V$. The regulated results are dominated by various power law divergences, where the powers depend on the spacetime dimension $d$. While these divergent terms have an interesting geometric character [6-8] e.g., the leading 'area law' contribution: $S_{n} \simeq c_{d-2} \mathcal{A}(\partial V) / \delta^{d-2}$, the corresponding coefficients depend on the details of the regulator. However, examining $S_{n}$ and $S_{\mathrm{EE}}$ in detail will also reveal universal contributions, whose coefficients are independent of the regulator and so provide unambiguous information about the underlying QFT. In particular, if the entangling surface is smooth, in an even number of dimensions, the universal contribution is characterized by a logarithmic divergence while in an odd number of dimensions, the constant contribution (i.e., $\delta$-independent term) will be universal if calculated with sufficient care [9]. That is,

$$
S_{n}^{\text {univ }}(V)= \begin{cases}(-1)^{\frac{d-1}{2}} s_{n}^{\text {univ }}(V) & d \text { odd } \\ (-1)^{\frac{d-2}{2}} s_{n}^{\text {univ }}(V) \log (R / \delta) & d \text { even }\end{cases}
$$




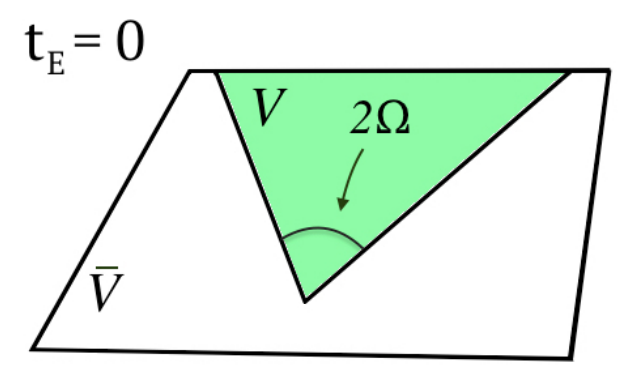

(a)

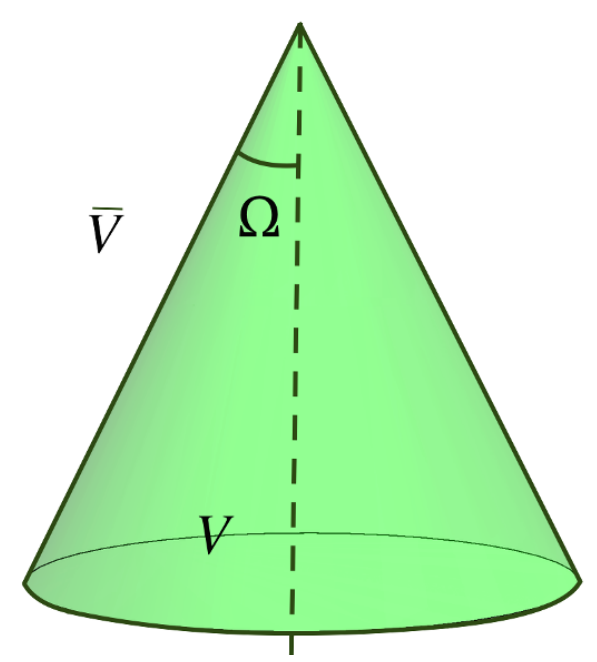

(b)

Figure 1. In panel (a), we show an entangling region $V$ whose boundary contains a sharp corner of opening angle $2 \Omega$. In panel (b), we show the analogous surface in $d=4$, i.e., a region whose boundary contains a conical singularity of opening angle $\Omega$. The smooth limit is found in both cases for $\Omega \rightarrow \pi / 2$.

where $R$ is some length scale characterizing the entangling region $V$. However, the situation changes when the entangling surface $\partial V$ contains geometric singularities. In particular, with a conical singularity, as illustrated in figure 1 , the regulator-independent terms take the form

$$
S_{n}^{\text {univ }}(V)= \begin{cases}(-1)^{\frac{d-1}{2}} a_{n}^{(d)}(\Omega) \log (R / \delta) & d \text { odd } \\ (-1)^{\frac{d-2}{2}} a_{n}^{(d)}(\Omega) \log ^{2}(R / \delta) & d \text { even }\end{cases}
$$

where $a_{n}^{(d)}(\Omega)$ are functions of the opening angle $\Omega$ of the cone. ${ }^{1}$ The appearance of a new logarithmic divergence associated with sharp corners in the entangling surface is well-known for the case of three dimensions [3-5, 13, 14]. The appearance of new universal terms in higher dimensions was first noted [8, 15] using holographic entanglement entropy [16-19]. Based on the latter results, our signs are chosen in eq. (1.3) to ensure that $a_{n}^{(d)}(\Omega) \geq 0$ for $0 \leq \Omega \leq \pi$ for all $d$.

Now if the entanglement or Rényi entropies are evaluated in a pure state, the results must be identical for the region $V$ and its complement $\bar{V}$. Therefore the corner function must satisfy

$$
a_{n}^{(d)}(\Omega)=a_{n}^{(d)}(\pi-\Omega)
$$

\footnotetext{
${ }^{1}$ Please notice that as illustrated in figure $1(\mathrm{a})$, the opening angle of a corner in $d=3$ is defined to be ' $2 \Omega$ ' in the present paper. This contrasts with our conventions in [10-12], where the same angle was called ' $\theta$ ' and ' $\Omega$,' respectively. The present convention simplifies the connection to higher-dimensional cones, for which we are using the same convention as in [8]. Notice, however, that the corner coefficient is defined in eq. (1.5) so that $\sigma_{n}^{(3)}$ agrees with the coefficients evaluated in [10-12] for three dimensions.
} 


\begin{tabular}{|c|c|c|c|c|c|c|c|c|}
\hline$d$ & 3 & 4 & 5 & 6 & 7 & 8 & 9 & 10 \\
\hline$\sigma^{(d)} / C_{\mathrm{T}}$ & $\frac{\pi^{2}}{24}$ & $\frac{\pi^{4}}{640}$ & $\frac{\pi^{4}}{270}$ & $\frac{\pi^{6}}{14336}$ & $\frac{\pi^{6}}{9450}$ & $\frac{5 \pi^{8}}{3538944}$ & $\frac{4 \pi^{8}}{2480625}$ & $\frac{7 \pi^{10}}{415236096}$ \\
\hline
\end{tabular}

Table 1. Cone coefficients $\sigma^{(d)}$ normalized by the stress tensor charge $C_{\mathrm{T}}$ in general holographic theories for various dimensions.

Further, our convention is that the entangling surface becomes smooth with $\Omega=\pi / 2$ and hence $a_{n}^{(d)}(\Omega=\pi / 2)=0$. Now assuming that these functions are smooth in the vicinity of $\Omega=\pi / 2$, these two results constrain the form of the cone functions with

$$
a_{n}^{(d)}(\Omega \rightarrow \pi / 2)=4 \sigma_{n}^{(d)}\left(\frac{\pi}{2}-\Omega\right)^{2},
$$

in general. Hence the universal corner contribution (1.3) defines a set of coefficients $\sigma_{n}^{(d)}$ which encode regulator-independent information about the underlying QFT.

While the above comments apply for general QFTs, we will focus on conformal field theories (CFTs) throughout the following. In refs. [10-12], we considered the properties of the corner coefficients $\sigma_{n}^{(3)}$ arising in three-dimensional CFTs. In particular, for the coefficient appearing in the EE, we argued that

$$
\sigma^{(3)} \equiv \sigma_{1}^{(3)}=\frac{\pi^{2}}{24} C_{\mathrm{T}},
$$

for general three-dimensional CFTs. That is, $\sigma^{(3)}$ is proportional to the central charge $C_{\mathrm{T}}$ appearing in the two-point correlator of the stress tensor. Evidence for this conjecture comes from free scalars and fermions, as well as certain holographic theories [10, 12, 20]. We extend the proof to general holographic theories in the present paper - see also [21] for an alternate approach. Of course, it is tempting to think about possible extensions of the above relation to higher dimensions. In fact, an analogous result is known for general CFTs in $d=4$ where [15]

$$
\sigma^{(4)}=\frac{\pi^{4}}{640} C_{\mathrm{T}} .
$$

In this paper, we prove that, at least for general holographic CFTs, $\sigma^{(d)}$ is indeed proportional to $C_{\mathrm{T}}$ in arbitrary dimensions. In particular, we find the general formula ${ }^{2}$

$$
\sigma^{(d)}=C_{\mathrm{T}} \frac{\pi^{d-1}(d-1)(d-2) \Gamma\left[\frac{d-1}{2}\right]^{2}}{8 \Gamma[d / 2]^{2} \Gamma[d+2]} \times \begin{cases}\pi & d \text { odd }, \\ 1 & d \text { even } .\end{cases}
$$

Hence, we conjecture that the cone coefficients $\sigma^{(d)}$ are related to $C_{\mathrm{T}}$ through eq. (1.8) for general CFTs. In table 1 , we show the values $\sigma^{(d)} / C_{\mathrm{T}}$ for $d=3,4, \cdots, 10$.

In [11], we proposed a generalization of eq. (1.6) to general Rényi entropies. According to this, the corresponding corner coefficients $\sigma_{n}^{(3)}$ are related to the scaling dimensions of the corresponding twist operators $h_{n}$ — see appendix A for definitions - through

$$
\sigma_{n}^{(3)}=\frac{1}{\pi} \frac{h_{n}}{n-1} .
$$

\footnotetext{
${ }^{2}$ Alternative proofs for general holographic theories in $d=3,4$ and 6 dimensions were presented in [21], using a different formalism. Some steps in this direction were also taken in [22].
} 
We have verified that eq. (1.9) is satisfied for all integer values of $n$ and in the limit $n \rightarrow \infty$ both for a free scalar and a free fermion [11] - see also [20, 23, 24]. Now all these results suggest a natural extension to the Rényi cone coefficients in higher dimensions. In particular, the expansion of the scaling dimension in general dimensions - see eq. (3.3) below - suggests that our result (1.8) extends to

$$
\sigma_{n}^{(d)}=\frac{h_{n}}{n-1} \frac{(d-1)(d-2) \pi^{\frac{d-4}{2}} \Gamma\left[\frac{d-1}{2}\right]^{2}}{16 \Gamma[d / 2]^{3}} \times \begin{cases}\pi & d \text { odd } \\ 1 & d \text { even } .\end{cases}
$$

We will show that this expression is consistent with previous results obtained for four- and six-dimensional CFTs [25, 26].

The remainder of the paper is organized as follows. In section 2, we use the results of [27] to prove our generalized conjecture (1.8) for general holographic theories. In section 1.1, we extend this conjecture to the Rényi cone coefficients (1.10) and use it to establish certain relations involving the structure of Rényi entropies for general entangling regions in four-dimensional theories. In section 4, we conclude with a brief discussion of our findings. Appendix A reviews some relevant information about twist operators and their conformal scaling dimensions.

\section{Cone coefficients for EE in general dimensions}

Our approach to proving eq. (1.8) is to take advantage of the results in [27] with regards to the leading correction to the entanglement entropy for a slightly deformed sphere $S^{d-2}$. In $[27,28]$, the authors consider the case in which $\partial V$ is a slightly deformed $S^{d-2}$ sphere of radius $R$ parametrized in polar coordinates as

$$
r\left(\Omega_{d-2}\right) / R=1+\epsilon \sum_{\ell, m_{1}, \ldots, m_{d-3}} a_{\ell, m_{1}, \ldots, m_{d-3}} Y_{\ell, m_{1}, \ldots, m_{d-3}}\left(\Omega_{d-2}\right)
$$

where $\epsilon$ is an infinitesimal parameter and $a_{\ell, m_{1}, \cdots, m_{d-3}}$ are some constant coefficients characterizing the deformation, Further, $Y_{l, m_{1}, \ldots, m_{d-3}}\left(\Omega_{d-2}\right)$ are (real) hyper-spherical harmonics ${ }^{3}$ on $S^{d-2}$ with coordinates $\Omega_{d-2}$. In particular, they are eigenfunctions of the Laplacian on the sphere with

$$
\triangle_{S^{d-2}} Y_{\ell, m_{1}, \ldots, m_{d-3}}\left(\Omega_{d-2}\right)=-\ell(\ell+d-3) Y_{\ell, m_{1}, \ldots, m_{d-3}}\left(\Omega_{d-2}\right)
$$

For such a deformed sphere, the coefficient in the universal part of the EE (1.2) takes the form

$$
s^{\text {univ }}(V)=s_{\text {sphere }}^{(d)}+\epsilon^{2} s_{2}^{(d)}(V)+\mathcal{O}\left(\epsilon^{3}\right) .
$$

\footnotetext{
${ }^{3}$ Here as in [27], the hyper-spherical harmonics are normalized such that:

$$
\int d \Omega_{d-2} Y_{\ell, m_{1}, \ldots, m_{d-3}} Y_{\ell^{\prime}, m_{1}^{\prime}, \ldots, m_{d-3}^{\prime}}=\delta_{\ell \ell^{\prime}} \delta_{m_{1} m_{1}^{\prime}} \cdots \delta_{m_{d-3} m_{d-3}^{\prime}} .
$$
}


Of course, the leading term here corresponds to the universal contribution for the undeformed sphere, which is given by $[29,30]$

$$
s_{\text {sphere }}^{(d)}= \begin{cases}F & d \text { odd } \\ 4 A & d \text { even } .\end{cases}
$$

In even dimensions, $A$ is precisely the coefficient appearing in the A-type trace anomaly while in odd dimensions, $F$ can be identified with the universal contribution in sphere partition function [31]. Of course, both of these coefficients are related to c-theorems in higher dimensions [29, 30, 32-34].

As shown in [28], the linear contribution in eq. (2.4) vanishes for a general CFT in arbitrary dimensions. The second order contribution was studied by Mezei in [27]. There he shows that for general holographic theories, this quadratic term is fully determined by the central charge $C_{\mathrm{T}}$ appearing in the two-point function of the stress tensor [35]:

$$
\left\langle T_{\mu \nu}(x) T_{\rho \sigma}(0)\right\rangle=\frac{C_{\mathrm{T}}}{x^{2 d}} \mathcal{I}_{\mu \nu, \rho \sigma}(x),
$$

where $\mathcal{I}_{\mu \nu, \rho \sigma}$ is a fixed dimensionless tensor. In particular, $s_{2}^{(d)}$ and $C_{\mathrm{T}}$ are related through the compact expression [27]

$$
s_{2}^{(d)}(V)=C_{\mathrm{T}} \frac{\pi^{\frac{d+2}{2}}(d-1)}{2^{d-2} \Gamma(d+2) \Gamma(d / 2)} \sum_{\ell, m_{1}, \ldots, m_{d-3}} a_{\ell, m_{1}, \ldots, m_{d-3}}^{2} \frac{\Gamma(d+\ell-1)}{\Gamma(\ell-1)} \times \begin{cases}\pi / 2 & d \text { odd }, \\ 1 & d \text { even } .\end{cases}
$$

Of course, this result clearly resembles our general conjecture (1.6) for $d=3$ CFTs: both $\sigma^{(3)}$ and $s_{2}^{(d)}$ are universal $\mathcal{O}\left(\epsilon^{2}\right)$ corrections to the EE of a smooth surface, (e.g., $(\Omega-$ $\pi / 2)^{2} \sim \epsilon^{2}$ with $\epsilon \ll 1$ as $\left.\Omega \rightarrow \pi / 2\right)$ and both coefficients are fully determined by $C_{\mathrm{T}}$. There are also some differences though: $\sigma^{(3)}$ characterizes a very particular deformation, namely the one which makes a sharp corner appear in the entangling surface, while $s_{2}^{(d)}$ encodes the contribution from a completely general smooth deformation of a hypersphere in general dimensions. On the other hand, as we have explained, the structure of EE divergences changes when the entangling surface $\partial V$ contains a conical singularity. In particular, the universal term for smooth surface is constant (logarithmically divergent) for odd (even) dimensional theories. However, that appearing with a conical singularity has a logarithmic (logarithmic $^{2}$ ) divergence for odd (even) $d$ - compare eq. (1.2) with eq. (1.3). Therefore, if $s_{2}^{(d)}$ is to capture the corner contribution $\sigma^{(3)}$ and its natural extensions to higher-dimensions $\sigma^{(d)}$, the corresponding calculation must involve the appearance of an extra logarithmic divergence $\log (R / \delta)$ in each case. ${ }^{4}$ We will see that this is indeed the case, and how by choosing particular deformations of $S^{d-2}$ which make infinitesimal conical singularities appear in the surface of the hypersphere, the corner coefficients $\sigma^{(d)}$ can be identified in general dimensions using eq. (2.7). At the same time, this approach provides a general holographic proof of our conjecture (1.6) and also extends it to higher dimensions as in eq. (1.8). We start below with the $d=3$ case, corresponding to our original conjecture (1.6).

\footnotetext{
${ }^{4}$ Constructions involving the appearance of analogous logarithmic contributions can be found e.g., in $[8,15]$.
} 


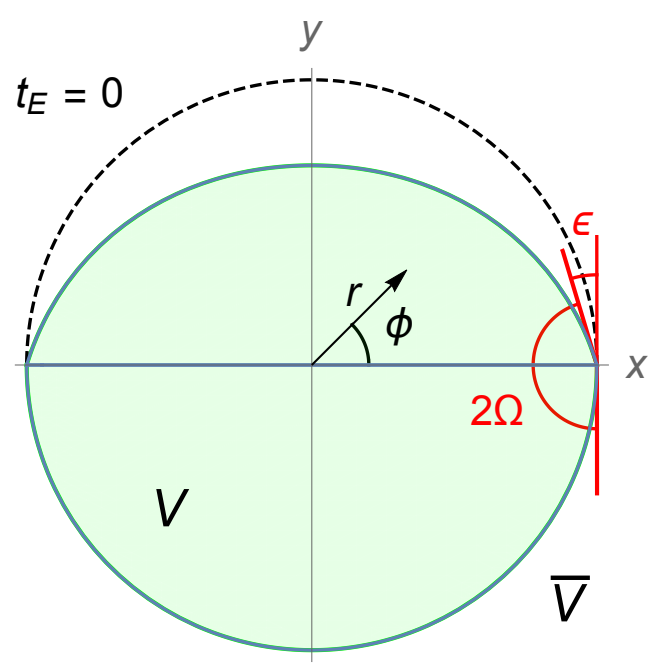

Figure 2. An entangling region $V$ whose boundary corresponds to a deformed $S^{1}$ in eq. (2.8). Two infinitesimal corner singularities appear at $\phi=0$ and $\pi$, with opening angle $2 \Omega=\pi-\epsilon$.

\subsection{Three dimensions}

Consider calculating the EE in a three-dimensional CFT for a deformed circular entangling surface. In particular, we consider the following deformation of the entangling surface

$$
r(\phi) / R= \begin{cases}1-\epsilon \sin \phi & \phi \in[0, \pi], \\ 1 & \phi \in(\pi, 2 \pi),\end{cases}
$$

parametrized by the polar coordinate $\phi \in[0,2 \pi)$ - see figure 2 . Below the $x$-axis, the circle remains undeformed but in the upper half plane, the surface is deformed in a way such that the surface is continuous for all values of $\phi$, but two infinitesimal corners or kinks are introduced at the $x$-axis, i.e., at $\phi=0, \pi$. It is straightforward to check that the deficit angle at both of these points is given by $\epsilon$ In particular, we see from eq. (2.8) that for $|\phi| \ll 1$, the position of the entangling surface in Euclidean coordinates is given by

$$
\begin{aligned}
& y=\phi R+\mathcal{O}\left(R \phi^{3}\right), \\
& x= \begin{cases}R-\epsilon R \phi+\mathcal{O}\left(R \phi^{2}\right) & \text { for } \phi \geq 0, \\
R+\mathcal{O}\left(R \phi^{2}\right) & \text { for } \phi \leq 0 .\end{cases}
\end{aligned}
$$

Hence, there is a kink in the entangling surface as it passes through the $x$-axis with $x \simeq$ $R-\epsilon y$ for $y \geq 0$ and $x \simeq R$ for $y \leq 0$. Further the slope of the tangent to the deformed surface for positive $\phi$ (with respect to the vertical) is precisely equal to $\epsilon$. This slope equals the tangent of the deficit angle and so we have: $\tan (\pi-2 \Omega) \simeq(\pi-2 \Omega) \simeq \epsilon$. Of course, a similar analysis of the second kink at $\phi=\pi$ yields the same deficit angle - see figure 2 .

Now our strategy is to 'Fourier transform' the deformation of the circular profile in eq. (2.8) so that it may be represented as in eq. (2.1). We may then evaluate the universal contribution to the EE using eq. (2.7). For $d=3$, an orthonormal basis of real polar 
harmonics is given by

$$
Y_{\ell}^{(c)}=\frac{1}{\sqrt{\pi}} \cos (\ell \phi), \quad Y_{\ell}^{(s)}=\frac{1}{\sqrt{\pi}} \sin (\ell \phi) .
$$

With these functions, the corresponding coefficients in eq. (2.1) become

$$
\begin{aligned}
& a_{\ell}^{(s)}=-\frac{1}{\sqrt{\pi}} \int_{0}^{\pi} \sin \phi \sin (\ell \phi) d \phi=-\left(\frac{\sin (\ell \pi)}{\sqrt{\pi}\left(1-\ell^{2}\right)}\right), \\
& a_{\ell}^{(c)}=-\frac{1}{\sqrt{\pi}} \int_{0}^{\pi} \sin \phi \cos (\ell \phi) d \phi=-\left(\frac{1+\cos (\ell \pi)}{\sqrt{\pi}\left(1-\ell^{2}\right)}\right) .
\end{aligned}
$$

However, the only non-vanishing components are:

$$
a_{1}^{(s)}=-\frac{\sqrt{\pi}}{2} \quad \text { and } \quad a_{2 k}^{(c)}=\frac{2}{\sqrt{\pi}\left(4 k^{2}-1\right)} \quad \text { for } \quad k=0,1,2, \cdots .
$$

Substituting these expressions into eq. (2.7), we find

$$
s_{2}^{(3)}(V)=\frac{\pi^{2} C_{\mathrm{T}}}{2} \sum_{k=1} \frac{k}{4 k^{2}-1} .
$$

For large $k$, the summand above is approximately $1 /(4 k)$ and so this sum is logarithmically divergent. If we regulate by cutting off the sum at $k=k_{\max }$, we find

$$
s_{2}^{(3)}(V)=\frac{\pi^{2} C_{\mathrm{T}}}{12}\left[\gamma-1+\log 4+\log \left(k_{\max }\right)+\mathcal{O}\left(1 / k_{\max }\right)\right],
$$

where $\gamma$ is the Euler-Mascheroni constant.

As discussed above, we anticipated the appearance of this new logarithmic divergence due to the presence of the two corner singularities in the deformed entangling surface (2.8). We can understand the divergent term physically as follows: we can think of $\ell$ in each Fourier mode as the physical (dimensionless) wavenumber of the corresponding perturbation of the circle. Hence, they can be associated with the corresponding wavelengths through $\lambda \sim 2 \pi R / \ell$. Now, we should truncate the sum in eq. (2.14) when these wavelengths are of the order of the UV cutoff, i.e., when $\ell \sim 2 \pi R / \delta$. Hence, it is natural to set $\ell_{\max }=2 k_{\max }=2 \pi R / \delta$ in eq. (2.15). This identification gives rise to exactly the desired logarithmic term required to match that appearing in the corner contribution to the EE. In particular, using eqs. (1.2) and (2.4), as well as $\epsilon=\pi-2 \Omega$, we find

$$
S^{\text {univ }}(V)=-\frac{\pi^{2}}{3} C_{\mathrm{T}}(\pi / 2-\Omega)^{2} \log (R / \delta) .
$$

Given this expression and recalling that this term contains two corner contributions, we find the expected result ${ }^{5}$

$$
\sigma^{(3)}=\frac{\pi^{2}}{24} C_{\mathrm{T}} .
$$

\footnotetext{
${ }^{5}$ Mark Mezei found the same result independently using a similar procedure. We thank him for discussions on his calculations.
} 


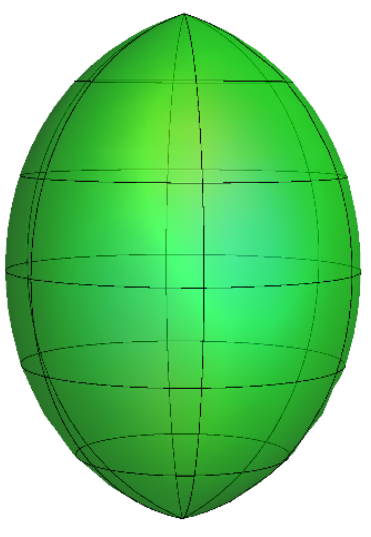

(a)

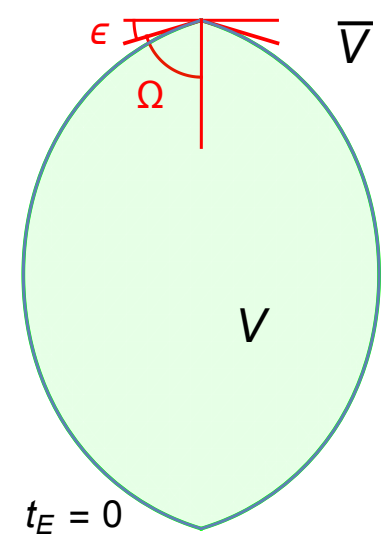

(b)

Figure 3. In panel (a), we show a two-sphere deformed according to eq. (2.18) with $\epsilon=0.3$. We observe the appearance of two conical singularities in the poles. In panel (b), we plot a cross-section of the same surface. The deficit angle of the conical singularities is determined by $\epsilon=\pi / 2-\Omega$ for small $\epsilon$.

The elegant result (2.7) of [27] was the essential ingredient in our discussion above. The analysis in [27] involves the expression for holographic entanglement entropy constructed in $[36,37]$ for bulk theories of higher derivative gravity, whose Lagrangian does not contain derivatives of the Riemann tensor. Hence the above discussion extends the proof of our original conjecture (1.6) to this (infinite) class of general holographic theories. Of course, it is very likely that eq. (2.7) applies for all three-dimensional CFTs, in which case the present discussion would provide a general proof of eq. (1.6).

\subsection{General higher-dimensional case}

Let us now turn to the higher-dimensional case. In particular, we consider a spherical entangling surface in a $d$-dimensional CFT with $d \geq 4$. We then make the following infinitesimal deformation of the sphere $S^{d-2}$,

$$
r\left(\Omega_{d-2}\right) / R=1-\epsilon \sin (\theta),
$$

where $\theta$ is the polar angle $\theta \in[0, \pi]$. With this deformation, the $S^{d-2}$ looks like a $(d-2)$ dimensional rugby ball, which we denote $R b^{d-2}-$ see figure $3(\mathrm{a})$. Hence, there are two conical singularities at the poles $\theta=0$ and $\pi$. The deformation parameter $\epsilon$ in eq. (2.18) determines the deficit angles at these singularities through $\epsilon=\pi / 2-\Omega-$ see figure 3(b). ${ }^{6}$

Now our analyis is simplified since $R b^{d-2}$ preserves an $\mathrm{SO}(d-2)$ subgroup of the $\mathrm{SO}(d-1)$ group of isometries of the round $S^{d-2}$. The Laplace operator on $S^{d-2}$ can be written recursively as ${ }^{7}$

$$
\triangle_{S^{d-2}}=\frac{1}{(\sin \theta)^{d-3}} \frac{\partial}{\partial \theta}\left[(\sin \theta)^{d-3} \frac{\partial}{\partial \theta}\right]+\frac{1}{\sin ^{2} \theta} \triangle_{S^{d-3}},
$$

\footnotetext{
${ }^{6}$ This relation is slightly different from our construction for $d=3$ in the previous section.

${ }^{7}$ We use the following set of angular coordinates: $\theta, \theta_{2}, \ldots, \theta_{d-3} \in[0, \pi]$ and $\phi \in[0,2 \pi)$.
} 
where we have used the label $\theta$ for the first polar coordinate in each case. To describe the $R b^{d-2}$ hypersurface, we need only consider the subset of hyperspherical harmonics, $Y_{\ell}(\theta)$, depending only on $\theta$, i.e., we concentrate on $m_{1}=\cdots=m_{d-3}=0$ from the general hyper-spherical harmonics. In this case, the Laplace equation (2.3) simplifies to

$$
\frac{1}{(\sin \theta)^{d-3}} \frac{\partial}{\partial \theta}\left[(\sin \theta)^{d-3} \frac{\partial}{\partial \theta}\right] Y_{\ell}(\theta)=-\ell(\ell+d-3) Y_{\ell}(\theta) \text {. }
$$

The general solution to eq. (2.20) is given by

$$
Y_{\ell}(\theta)=\frac{p_{\ell}}{\sin ^{(d-4) / 2} \theta} P_{\ell+(d-4) / 2}^{(d-4) / 2}(\cos \theta)+\frac{q_{\ell}}{\sin ^{(d-4) / 2} \theta} Q_{\ell+(d-4) / 2}^{(d-4) / 2}(\cos \theta),
$$

where $P_{\ell}^{m}(x)$ and $Q_{\ell}^{m}(x)$ are associated Legendre polynomials (ALPs) of the first and second kind, respectively, and where $p_{\ell}$ and $q_{\ell}$ are normalization constants, which will be fixed by demanding $\int d \Omega_{d-2} Y_{\ell}(\theta) Y_{\ell^{\prime}}(\theta)=\delta_{\ell \ell^{\prime}}$. In even dimensions, the ALPs of the second kind are generically non-normalizable and so we set $q_{\ell}=0$ in this case. ${ }^{8}$ Similarly, in odd dimensions, the ALPs of the first kind that are non-normalizable and we must set $p_{\ell}=0$. Hence we find:

$$
\begin{aligned}
d \text { even }: & p_{\ell}=\hat{p}_{\ell}, & q_{\ell}=0 ; \\
d \text { odd }: & p_{\ell}=0, & q_{\ell}=\frac{2}{\pi} \hat{p}_{\ell} .
\end{aligned}
$$

where

$$
\hat{p}_{\ell}^{2} \equiv \frac{\Gamma[\ell+1]}{\Gamma[d+\ell-3]} \frac{(d+2 \ell-3)}{2 \Omega_{d-3}} \quad \text { with } \quad \Omega_{d-3}=\frac{2 \pi^{(d-2) / 2}}{\Gamma[(d-2) / 2]},
$$

which is the volume of a unit $S^{d-3}$.

Hence with the deformation in eq. (2.18), we evaluate the coefficients in the expansion $(2.1)$ as

$$
\begin{aligned}
a_{\ell} & =q_{\ell} \sqrt{\Omega_{d-3}} \int_{0}^{\pi} d \theta \sin ^{d / 2} \theta Q_{\ell+(d-4) / 2}^{(d-4) / 2}(\cos \theta), & & d \text { odd } ; \\
& =p_{\ell} \sqrt{\Omega_{d-3}} \int_{0}^{\pi} d \theta \sin ^{d / 2} \theta P_{\ell+(d-4) / 2}^{(d-4) / 2}(\cos \theta), & & d \text { even } .
\end{aligned}
$$

Now for both odd and even dimensions, we find that $a_{\ell}^{2}$ are only nonvanishing for even values of $\ell$, i.e., $\ell=2 k$. Interestingly, the final value can be expressed in a closed form valid for all dimensions $d \geq 4$, odd or even, which reads

$$
a_{2 k}^{2}=\frac{(4 k+d-3) \pi^{d / 2-2} \Gamma\left[\frac{d-1}{2}\right]^{2} \Gamma\left[k-\frac{1}{2}\right]^{2} \Gamma\left[k+\frac{1}{2}\right] \Gamma\left[k+\frac{d-3}{2}\right]}{4 \Gamma\left[\frac{d-2}{2}\right] \Gamma[k+1] \Gamma\left[k+\frac{d}{2}-1\right] \Gamma\left[k+\frac{d}{2}\right]^{2}} .
$$

We can now use this expression in Mezei's formula (2.7) to find

$$
s_{2}^{(d)}(V)=C_{\mathrm{T}} \frac{\pi^{\frac{d+2}{2}}(d-1)}{2^{d-2} \Gamma(d+2) \Gamma(d / 2)} \sum_{k} A_{k} \times \begin{cases}\pi / 2 & d \text { odd }, \\ 1 & d \text { even },\end{cases}
$$

\footnotetext{
${ }^{8}$ For example, for $d=4$, we will have: $Y_{\ell}(\theta)=\sqrt{\frac{(2 \ell+1)}{4 \pi}} P_{\ell}(\cos \theta)$, which are the usual zonal harmonics on $S^{2}$.
} 
where

$$
A_{k}=\frac{(4 k+d-3) \pi^{d / 2-2} \Gamma\left[\frac{d-1}{2}\right]^{2} \Gamma\left[k-\frac{1}{2}\right]^{2} \Gamma\left[k+\frac{1}{2}\right] \Gamma\left[k+\frac{d-3}{2}\right]}{4 \Gamma\left[\frac{d-2}{2}\right] \Gamma[k+1] \Gamma\left[k+\frac{d}{2}-1\right] \Gamma\left[k+\frac{d}{2}\right]^{2}} \frac{\Gamma(2 k+d-1)}{\Gamma(2 k-1)} .
$$

Now the behaviour of $A_{k}$ as $k \rightarrow \infty$ is given by

$$
A_{k}=\frac{2^{d} \pi^{d / 2-2} \Gamma\left[\frac{d-1}{2}\right]^{2}}{\Gamma\left[\frac{d-2}{2}\right]} \frac{1}{k}+\mathcal{O}\left(1 / k^{2}\right)
$$

and so eq. (2.26) becomes

$$
s_{2}^{(d)}(V)=C_{\mathrm{T}} \frac{\pi^{\frac{d+2}{2}}(d-1)}{2^{d-2} \Gamma(d+2) \Gamma(d / 2)}\left[\frac{2^{d} \pi^{d / 2-2} \Gamma\left[\frac{d-1}{2}\right]^{2}}{\Gamma\left[\frac{d-2}{2}\right]} \log \left(k_{\max }\right)+\cdots\right] \times \begin{cases}\pi / 2 & d \text { odd }, \\ 1 & d \text { even },\end{cases}
$$

where again we have introduced a cut-off $k_{\max }$, and the ellipsis refers to terms which do not scale with $k_{\max }$ (or with inverse powers of this cut-off). Hence we can see that the two conical singularities at the poles of the sphere again give rise to an additional logarithmic divergence in the EE. Comparing to eq. (1.2), the universal contribution to the EE becomes

$$
S^{\text {univ }}(V)=C_{\mathrm{T}} \frac{2(d-1)(d-2) \pi^{d-1} \Gamma\left[\frac{d-1}{2}\right]^{2}}{\Gamma\left[\frac{d}{2}\right]^{2} \Gamma[d+2]} \epsilon^{2} \times \begin{cases}(-1)^{\frac{d-1}{2}} \pi / 2 \log \left(k_{\max }\right) & d \text { odd, } \\ (-1)^{\frac{d-2}{2}} \log \left(k_{\max }\right) \log (R / \delta) & d \text { even. }\end{cases}
$$

This expression above contains contributions from two conical singularities (one at each pole of the sphere) and so we must divide by 2 in order to extract $\sigma^{(d)}$ in all cases. In odd dimensions, we replace $\log \left(k_{\max }\right)=\log (\pi R / \delta)$, just like for $d=3$, which allows us to identify

$$
\sigma^{(d)}=C_{\mathrm{T}} \frac{(d-1)(d-2) \pi^{d} \Gamma\left[\frac{d-1}{2}\right]^{2}}{8 \Gamma\left[\frac{d}{2}\right]^{2} \Gamma[d+2]}, \quad d \text { odd } .
$$

We show some explicit values in table 1 . Note that this formula for general odd $d$ properly incorporates the result (1.6) for $d=3$. Interestingly, the analytic values given here for $d \geq 5$ are not easily accessible through the standard calculation using the Ryu-Takayanagi (RT) prescription [16-19] for an entangling surface containing a conical singularity. In particular, the corresponding corner functions $a^{(5,7, \cdots)}(\Omega)$ of the opening angle are given by complicated implicit expressions which can only be treated numerically, e.g., see [8] for a discussion of the $d=5$ case.

In even dimensions, there is a subtlety in the identification of $\log \left(k_{\max }\right)$ with $\log (R / \delta)$, which requires comment below. Indeed, in this case, the correct substitution is $\log \left(k_{\max }\right)=$ $\frac{1}{2} \log (\pi R / \delta)$ instead. Taking this into account, we find

$$
\sigma^{(d)}=C_{\mathrm{T}} \frac{(d-1)(d-2) \pi^{d-1} \Gamma\left[\frac{d-1}{2}\right]^{2}}{8 \Gamma\left[\frac{d}{2}\right]^{2} \Gamma[d+2]}, \quad d \text { even } .
$$

We have explicitly verified that this formula exactly reproduces the results obtained using the RT prescription for a cone of opening angle $\Omega=\pi / 2-\epsilon$ for $d=4,6,8,10,12,14$ - see table 1. 
Let us now comment on the reason behind the factor $1 / 2$ which appears above in the even-dimensional case when relating the highest wavenumber $k_{\max }$ to the short distance cutoff $\delta .{ }^{9}$ An illustrative way of understanding this factor consists of comparing our calculation here with the one performed using the RT prescription for an entangling region consisting of a cone (e.g., in $d=4)$. In the latter, the $\log (R / \delta)^{2}$ term arises from an integral of the form $\int_{R}^{\delta} \frac{d r}{r} \log (r / \delta)=\frac{1}{2} \log (R / \delta)^{2}$, e.g., see [8]. In our present calculation, the two logarithms arise separately. One is a fixed overall factor in the universal term (1.2) of a generic smooth surface in even dimensions. The other is obtained from the sum $\sum_{k=1}^{k_{\max }} 1 / k$, which produces the $\log \left(k_{\max }\right)$ factor. The latter is like performing the integration $\int_{R}^{\delta} \frac{d r}{r}$ separate from the $\operatorname{logarithmic}$ factor and we observe that the naive substitution $\log \left(k_{\max }\right) \rightarrow \log (R / \delta)$ fails to yield the correct answer by precisely a factor 2 [15]. This holographic calculation suggests that there should not be a fixed infrared scale in the overall logarithmic factor appearing in the universal term (1.2). At least for the perturbations at smaller wavelengths, this IR scale should match the wavelength of the perturbation. More pragmatically, in order to produce the correct $\frac{1}{2} \log (R / \delta)^{2}$ factor in our calculation, we need to make the replacement $\log \left(k_{\max }\right) \rightarrow \frac{1}{2} \log (R / \delta)$ instead. From this discussion, it is also clear that the subtlety is exclusive to theories in even dimensions since in odd dimensions, the analogous integral in the RT calculation is of the form $\int_{R}^{\delta} \frac{d r}{r}=\log (R / \delta)$, and therefore it does not produce any additional factor with respect to our naive substitution $\log \left(k_{\max }\right) \rightarrow \log (R / \delta) .{ }^{10} \mathrm{In}$ particular, we have seen that the $d=3$ calculation gives rise to the correct $\sigma^{(3)} / C_{\mathrm{T}}$ ratio.

With this explanation we conclude our proof of the relation $\sigma^{(d)} / C_{\mathrm{T}}$ in general dimensions for general holographic theories, which is summarized in eq. (1.8). Again, if eq. (2.7) holds for general CFTs in arbitrary dimensions, our results here would provide a general proof of eq. (1.8). In any event, we conjecture that eq. (1.8) applies not only for holographic CFTs but for general CFTs.

\section{Cone coefficients for Rényi entropy}

A conical defect in an otherwise smooth entangling surface will introduce a new universal contributions to the Rényi entropy (1.3), analogous to those discussed above for the EE. In particular, the corresponding Rényi cone contributions $a_{n}^{(d)}(\Omega)$ behave as in eq. (1.5) for large opening angles. In [11], we considered the corresponding corner coefficient $\sigma_{n}^{(3)}$ controlling this universal contribution to the Rényi entropy for an almost smooth entangling surface. There, we argued that our original conjecture (1.6) is a particular case of a more general relation connecting $\sigma_{n}^{(3)}$ with the scaling dimension of the corresponding twist operators $h_{n}$ with

$$
\sigma_{n}^{(3)}=\frac{1}{\pi} \frac{h_{n}}{n-1}
$$

\footnotetext{
${ }^{9}$ The very same factor $1 / 2$ was observed to appear in $[8,15]$ and $[26]$ when computing $a^{(d)}(\Omega)$ for $d=4$ and 6 , respectively.

${ }^{10}$ We omitted the factor $\pi$ in the relation $k_{\max } \sim \pi R / \delta$ in this paragraph as it does not play any role in the final result and it might however be confusing.
} 
In [11], we verified that eq. (3.1) is satisfied for all integer values of $n$ and in the limit $n \rightarrow \infty$ both for a free scalar and a free fermion - see also [23, 24].

While we provide a precise definition of the scaling dimension $h_{n}$ in appendix A, a key result for our present purposes will be $[38,39]^{11}$

$$
\left.\partial_{n} h_{n}\right|_{n=1}=2 \pi^{\frac{d+2}{2}} \frac{\Gamma[d / 2]}{\Gamma[d+2]} C_{\mathrm{T}} .
$$

That is, that the first derivative of the scaling dimension at $n=1$ is determined by the central charge $C_{\mathrm{T}}$. Recalling that $h_{1}=0$, we may use eq. (3.2) to write the leading term in an expansion about $n=1$ as,

$$
h_{n} \stackrel{n \rightarrow 1}{=} 2 \pi^{\frac{d+2}{2}} \frac{\Gamma[d / 2]}{\Gamma[d+2]} C_{\mathrm{T}}(n-1)+\mathcal{O}\left((n-1)^{2}\right)
$$

In particular then, for $d=3$, we have $h_{n} \simeq \frac{\pi^{3}}{24} C_{\mathrm{T}}(n-1)$ and upon substituting this expansion into eq. (3.1), we see that it reduces to our original conjecture (1.6) for the EE at $n=1$. Therefore, the new conjecture (3.1) is supported by all of the evidence supporting eq. (1.6), including calculations for free scalars and fermions [10, 12, 20, 23, 24], as well as the general proof for holographic theories given above - see also [21].

Now these results suggest a natural extension to the Rényi cone coefficients in higher dimensions. In particular, the expansion (3.3) of the scaling dimension in general dimensions suggests that our result (1.8) extends to

$$
\sigma_{n}^{(d)}=\frac{h_{n}}{n-1} \frac{(d-1)(d-2) \pi^{\frac{d-4}{2}} \Gamma\left[\frac{d-1}{2}\right]^{2}}{16 \Gamma[d / 2]^{3}} \times \begin{cases}\pi & d \text { odd }, \\ 1 & d \text { even } .\end{cases}
$$

Hence for the next few dimensions, eq. (3.1) is supplemented by

$$
\sigma_{n}^{(4)}=\frac{3 \pi}{32} \frac{h_{n}}{n-1}, \quad \sigma_{n}^{(5)}=\frac{16}{9} \frac{h_{n}}{n-1}, \quad \sigma_{n}^{(6)}=\frac{45 \pi^{2}}{512} \frac{h_{n}}{n-1} .
$$

Now the scaling dimension of twist operators in the free scalar or free fermion theories can be straightforwardly calculated using heat kernel techniques in any number of dimensions [39] - see also [23, 24]. Hence it would be interesting if one could directly evaluate the coefficients $\sigma_{n}^{(d)}$ for these theories to provide further evidence supporting eq. (3.4). In the following, we take some steps in this direction for the cases of $d=4$ and $d=6$.

\subsection{Four dimensions}

The universal contribution to the Rényi entropy of a CFT for a general region can be expressed in terms of a geometric integral over the entangling surface [41] ${ }^{12}$

$$
S_{n}^{\text {univ }}=-\frac{\log (R / \delta)}{2 \pi} \int_{\partial V} d^{2} y \sqrt{h}\left[f_{a}(n) \mathcal{R}+f_{b}(n)\left(\operatorname{Tr} K^{2}-\frac{1}{2} K^{2}\right)-f_{c}(n) C_{a b}{ }^{a b}\right]
$$

\footnotetext{
${ }^{11}$ Ref. [40] considers an analogous derivative of the Rényi entropy.

${ }^{12}$ In this expression, $h_{a b}$ is the induced metric on the two-dimensional entangling surface $\partial V$ and $\mathcal{R}$ is the corresponding (intrinsic) Ricci scalar. The extrinsic curvature is denoted by $K_{a b}^{i}$ where $a, b$ and $i$ denote the two tangent directions and the two transverse directions to $\partial V$, respectively. Hence $\operatorname{Tr} K^{2} \equiv K_{a}^{i b} K_{b}^{i a}$ and $K^{2} \equiv K_{a}^{i a} K_{b}^{i b}$ where the indices are raised with the inverse metric $h^{a b}$. Further, $C_{a b}^{a b}$ is the background Weyl curvature also traced with $h^{a b}$.
} 
where the functions $f_{a, b, c}(n)$ are independent of the geometry of the entangling surface or the background spacetime. In the limit $n \rightarrow 1$, these functions are related to the coefficients appearing in the trace anomaly: ${ }^{13}$

$$
f_{a}(n=1)=a, \quad f_{b}(n=1)=c=f_{c}(n=1),
$$

and with these values, eq. (3.6) reduces to the corresponding expression for the universal contribution to the EE in four-dimensional CFTs [42].

Further, two of these coefficients are readily calculated for a massless free real scalar or a massless free Dirac fermion [41, 43-47]:

$$
\begin{aligned}
f_{a}^{\text {scalar }}(n) & =\frac{(1+n)\left(1+n^{2}\right)}{1440 n^{3}}, & f_{c}^{\text {scalar }}(n) & =\frac{(1+n)\left(1+n^{2}\right)}{480 n^{3}}, \\
f_{a}^{\text {fermion }}(n) & =\frac{(1+n)\left(7+37 n^{2}\right)}{2880 n^{3}}, & f_{c}^{\text {fermion }}(n) & =\frac{(1+n)\left(7+17 n^{2}\right)}{960 n^{3}} .
\end{aligned}
$$

Ref. [41] conjectured that the following relation held for all four-dimensional CFTs

$$
f_{b}(n)=f_{c}(n),
$$

and they provided numerical evidence for the free scalars and fermions that these two coefficients were identical. Further support was provided by [25], which argued that it also held for free Maxwell fields, $\mathcal{N}=4$ super-Yang-Mills and a broad class of holographic CFTs. Ref. [25] also argued that in general, $f_{a}(n)$ and $f_{c}(n)$ are related with

$$
f_{c}(n)=n\left(\frac{a-f_{a}(n)}{n-1}-\partial_{n} f_{a}(n)\right) .
$$

Now we may apply these results to determine the universal contribution to the Rényi entropy coming from a conical entangling surface in $d=4 .{ }^{14}$ Parametrizing the cone in spherical coordinates $\left(t_{E}, r, \theta, \phi\right)$ as $t_{E}=0, \theta=\Omega$ - see figure $1(\mathrm{~b})$ - it is easy to find the two normal vectors $n^{1}=\partial_{t_{E}}, n^{2}=r \partial_{\theta}$. The only non-vanishing component of the extrinsic curvatures associated to these vectors is $K_{\phi \phi}^{2}=1 / 2 r \sin 2 \Omega$. Using this result in eq. (3.6), one finds that the only nonvanishing contribution to the universal term (3.6) comes from the term proportional to $f_{b}(n)$

$$
S_{n}^{\text {univ }}=-\frac{1}{2} f_{b}(n) \frac{\cos ^{2} \Omega}{\sin \Omega} \log (R / \delta) \int_{r_{\min }}^{r_{\max }} \frac{d r}{r},
$$

where we have introduced UV and IR cut-offs in the radial integral, $r_{\min }$ and $r_{\max }$, respectively. As discussed in the previous section, the naive replacement $\log \left(r_{\max } / r_{\min }\right) \rightarrow$ $\log (R / \delta)$ fails to give the right answer by a factor 2 . Taking this into account, we find the correct result is

$$
S_{n}^{\text {univ }}=-\frac{1}{4} f_{b}(n) \frac{\cos ^{2} \Omega}{\sin \Omega} \log ^{2}(R / \delta) .
$$

\footnotetext{
${ }^{13}$ Note that we have adopted a convention where for a massless free real scalar field these coefficients are given by: $a=1 / 360$ and $c=1 / 120$. This may be contrasted with the conventions of [41] where the corresponding coefficients are given by: $a=1=c$.

${ }^{14}$ We closely follow the analogous derivation for the entanglement entropy, i.e., $n=1$, in [15].
} 
Note that this calculation has fixed the entire angular dependence of the cone contribution with

$$
a_{n}^{(4)}(\Omega)=\frac{1}{4} f_{b}(n) \frac{\cos ^{2} \Omega}{\sin \Omega} .
$$

Of course, this function exhibits the appropriate behaviour in the limit $\Omega \rightarrow \pi / 2$ given in eq. (1.5). In particular, we have

$$
\sigma_{n}^{(4)}=\frac{1}{16} f_{b}(n)
$$

Finally combining the above expression with our conjecture, we arrive at

$$
f_{b}(n)=\frac{3 \pi}{2} \frac{h_{n}}{n-1} .
$$

Now, the scaling dimension $h_{n}$ has been evaluated using heat kernel techniques for the free real scalar or free Dirac fermion theories in four dimensions as [39]

$$
\begin{aligned}
h_{n}^{\text {scalar }} & =\frac{1}{720 \pi} \frac{n^{4}-1}{n^{3}}, \\
h_{n}^{\text {fermion }} & =\frac{1}{1440 \pi} \frac{\left(n^{2}-1\right)\left(7+17 n^{2}\right)}{n^{3}},
\end{aligned}
$$

which combined with eq. (3.15) yields

$$
\begin{aligned}
f_{b}^{\text {scalar }}(n) & =\frac{(1+n)\left(1+n^{2}\right)}{480 n^{3}}, \\
f_{b}^{\text {fermion }}(n) & =\frac{(1+n)\left(7+17 n^{2}\right)}{960 n^{3}} .
\end{aligned}
$$

For these free CFTs, we know that eq. (3.9) certainly applies and hence we can compare these results to the expressions for $f_{c}(n)$ in eq. (3.8). We find complete agreement with those expressions and hence we have additional support for our conjecture (3.4).

We should note that by independent calculations, ref. [25] derived

$$
f_{c}(n)=\frac{3 \pi}{2} \frac{h_{n}}{n-1},
$$

as a general result for four-dimensional CFTs. This result then connects the two conjectures in eqs. (3.4) and (3.9). That is, finding a general proof of the four-dimensional version of our conjecture for the Rényi cone coefficient will provide a general proof of eq. (3.9) and vice versa. More generally if we accept both eqs. (3.9) and (3.10) for general fourdimensional CFTs, our calculations here indicate that all three of the coefficients in eq. (3.6) are completely determined by the scaling dimension of the twist operator (as well as the A-type trace anomaly coefficient). In particular, we find [25]

$$
\begin{aligned}
\partial_{n}\left[(n-1) f_{a}(n)\right] & =a-\frac{3 \pi}{2 n} h_{n} . \\
f_{b}(n) & =f_{c}(n)=\frac{3 \pi}{2} \frac{h_{n}}{n-1} .
\end{aligned}
$$


As an example, eq. (3.19) can be used to predict these coefficients for strongly coupled holographic CFTs dual to Einstein gravity [25]. In particular, we begin with the AdS/CFT correspondence in its simplest setting, where it describes a four-dimensional boundary CFT in terms of five-dimensional Einstein gravity in the bulk with the action

$$
I=\frac{1}{16 \pi G} \int d^{5} x \sqrt{g}\left[\frac{12}{L^{2}}+\mathcal{R}\right]
$$

where $G$ is the four-dimensional Newton's constant, $L$ is the $\mathrm{AdS}_{5}$ radius, and $\mathcal{R}$ is the Ricci scalar. In order to obtain $h_{n}^{\text {hol }}$, we need to consider the thermal ensemble of the boundary CFT on the hyperbolic geometry appearing in the construction of [31], which is then equivalent to a topological black hole with a hyperbolic horizon. We refer the interested reader to [38] for the detailed calculations and simply quote the result here:

$$
h_{n}^{\mathrm{hol}}=\frac{L^{3}}{G} \frac{8 n^{4}-4 n^{2}-1-\sqrt{1+8 n^{2}}}{256 n^{3}} .
$$

Of course, we also need the central charge $a$ for the boundary CFT $[48,49]: a^{\text {hol }}=\frac{\pi L^{3}}{8 G}$. Now, using the identities in eq. (3.19), we can easily use the above expression to compute the $f_{a, b, c}(n)$ coefficients. We find

$$
\begin{aligned}
& f_{a}^{\text {hol }}(n)=\frac{\pi L^{3}}{8 G}-\frac{\pi L^{3}}{128 G} \frac{1}{n^{3}}\left(6 n^{3}-10 n^{2}-n-1+\frac{2(n+1)\left(8 n^{2}+1\right)}{3+\sqrt{8 n^{2}+1}}\right) \\
& f_{b}^{\text {hol }}(n)=f_{c}^{\text {hol }}(n)=\frac{3 \pi L^{3}}{128 G} \frac{n+1}{n^{3}}\left(2 n^{2}+1-\frac{2}{3+\sqrt{8 n^{2}+1}}\right) .
\end{aligned}
$$

From these expressions, it is not difficult to verify that $f_{a}^{\text {hol }}(n=1)=a^{\text {hol }}=\pi L^{3} /(8 G)$, $f_{b}^{\text {hol }}(n=1)=f_{c}^{\text {hol }}(n=1)=c^{\text {hol }}=\pi L^{3} /(8 G)$, as expected.

\subsection{Six dimensions}

As we pointed out in the previous section, $a^{(4)}(\Omega)$ is proportional to $C_{\mathrm{T}}$ on general grounds for all values of $\Omega$ [15]. However, this behaviour seems to be particular for four-dimensional CFTs (and, of course, also trivially for $d=2$ theories). For example, for six-dimensional holographic CFTs which are dual to Gauss-Bonnet gravity in the bulk, one finds [8]

$$
a^{(6)}(\Omega)=\frac{3}{1024} \frac{\cos ^{2} \Omega}{\sin \Omega}\left(\left(\frac{5 \pi^{6}}{56} C_{\mathrm{T}}+3 A\right)-\left(\frac{\pi^{6}}{168} C_{\mathrm{T}}-3 A\right) \cos 2 \Omega\right),
$$

where $A$ is the universal coefficient of the A-type trace anomaly, i.e., the coefficient appearing in the universal EE of a spherical entangling surface, as in eq. (2.5). However, in the limit $\Omega \rightarrow \pi / 2$, the dependence on $A$ cancels in the leading contribution (1.5) and one finds $\sigma^{(6)} / C_{\mathrm{T}}=\pi^{6} / 14336$, as expected from eq. (1.8).

Interestingly, using the results in $[25,26]$, we can readily extend the validity of eqs. (1.8) and (1.10) for $d=6$ to a class of CFTs which extends beyond holographic theories. The idea is as follows: the universal term in the Rényi entropy for a general six-dimensional CFT can be written [26], in a analogous way to eq. (3.11) for $d=4$, with a sum of terms 
involving various combinations of intrinsic and extrinsic curvature tensors integrated over the entangling surface $\partial V$. For the EE (i.e., $n=1$ ), each of these combinations is weighted by one of the Weyl anomaly coefficients $A, B_{1}, B_{2}, B_{3}$

$$
\left\langle T^{\mu}{ }_{\mu}\right\rangle=\sum_{i=1}^{3} B_{i} I_{i}+2 A E_{6},
$$

where $E_{6}$ is the Euler density of six-dimensional manifolds and the $I_{i}$ are independent invariants consisting of various contractions of the Weyl tensor [50, 51]. In [26], it was shown that for all theories satisfying

$$
3 B_{3}=B_{2}-\frac{B_{1}}{2},
$$

the corresponding universal term (in a flat background) can be written as

$$
S^{\text {univ }}=\log (R / \delta) \int_{\partial V} d^{4} y \sqrt{h}\left[2 A E_{4}+6 \pi\left(B_{2}-\frac{B_{1}}{4}\right) J+B_{3} T_{3}\right],
$$

where $E_{4}$ is the four-dimensional Euler density, while $J$ and $T_{3}$ are certain complicated combinations of extrinsic curvatures. ${ }^{15}$ In theories satisfying eq. (3.25), eq. (3.26) can then be used to determine $\sigma^{(6)}$ along the lines of the previous discussion for $d=4$. The final result is $[26]$

$$
\sigma^{(6)}=\frac{27 \pi^{3}}{2} B_{3}
$$

Further, in [25], it was proven that $B_{3}$ and $C_{\mathrm{T}}$ are related for general theories through

$$
B_{3}=\frac{\pi^{3}}{193536} C_{\mathrm{T}} .
$$

Combining these two results, one then finds $\sigma^{(6)} / C_{\mathrm{T}}=\pi^{6} / 14336$, in agreement with our general formula eq. (1.8).

Let us add that the holographic CFTs dual to Gauss-Bonnet gravity satisfy the constraint in eq. (3.25) [26]. Hence eq. (3.23) provides the universal contribution from a conical entangling surface in $d=6$ for all angles in this class of theories. That is, when we apply eq. (3.26) to evaluate the universal contribution from a conical entangling surface in $d=6$ and we use eq. (3.28) to replace $B_{3}$ with $C_{\mathrm{T}}$, the final result matches that in eq. (3.23).

Hence, we find that all six-dimensional CFTs satisfying eq. (3.25) respect our new formula (1.8). As shown in [26], these include at least some holographic theories like Lovelock gravity, but also other theories, such as the interacting $\mathcal{N}=(2,0)$ theory describing a large number of coincident M5-branes, or a free $\mathcal{N}=(1,0)$ hypermultiplet consisting of one Weyl fermion and 4 real scalars. Interestingly, this is the minimal free model for which eq. (3.25)

\footnotetext{
${ }^{15}$ In particular, they are given by $J \equiv \frac{5 K^{2}}{4}\left(\frac{K^{2}}{8}-\operatorname{Tr} K^{2}\right)+\left(\operatorname{Tr} K^{2}\right)^{2}+2\left(K \operatorname{Tr} K^{3}-\operatorname{Tr} K^{4}\right)$ and $T_{3} \equiv$ $\left(\nabla_{a} K\right)^{2}-\frac{25}{16} K^{4}+11 K^{2} \operatorname{Tr} K^{2}-6\left(\operatorname{Tr} K^{2}\right)^{2}-16 K \operatorname{Tr} K^{3}+12 \operatorname{Tr} K^{4}$ respectively.
} 
is fulfilled, e.g., a single free scalar or a free fermion do not satisfy eq. (3.25). Indeed, the anomaly coefficients for a free real scalar and a free Dirac fermion read [50]

$$
\begin{array}{ll}
B_{1}^{\text {scalar }}=-\frac{1}{(4 \pi)^{3} 540}, & B_{1}^{\text {fermion }}=-\frac{8}{(4 \pi)^{3} 135}, \\
B_{2}^{\text {scalar }}=\frac{1}{(4 \pi)^{3} 3024}, & B_{2}^{\text {fermion }}=-\frac{2}{(4 \pi)^{3} 315}, \\
B_{3}^{\text {scalar }}=\frac{1}{(4 \pi)^{3} 2520}, & B_{3}^{\text {fermion }}=\frac{1}{(4 \pi)^{3} 126} .
\end{array}
$$

Hence the constraint eq. (3.25) is only satisfied when the theory contains 4 real scalars for each Weyl fermion, as in the $\mathcal{N}=(1,0)$ hypermultiplet. As a result, we do not yet have a proof of our general conjecture eq. (1.8) for free fields in $d=6$.

The previous discussion can be extended to general values of the Rényi index $n$. In that case, the coefficients $B_{i}$ are replaced by certain functions $f_{B_{i}}(n)$ satisfying $f_{B_{i}}(n=1)=B_{i}$. In [25], it was shown that $f_{B_{3}}(n)$ and the scaling dimension $h_{n}$ are related for general theories through

$$
f_{B_{3}}(n)=\frac{5}{768 \pi} \frac{h_{n}}{n-1} .
$$

Now we can try to extend the calculation of $\sigma^{(6)}$ in [26] to general Rényi entropies. In particular, it is natural to expect that eq. (3.26) extends to general Rényi entropies by simply replacing $B_{i}$ by the $f_{B_{i}}(n)$ (and $A$ by some $f_{A}(n)$ ) for theories satisfying some constraint $F\left(f_{B_{i}}(n)\right)=0$. This constraint should be such that it reduces to eq. (3.25) for $n=1$. Hence, it is natural to expect a relation of the form

$$
3 f_{B_{3}}(n)=f_{B_{2}}(n)-\frac{f_{B_{1}}(n)}{2} .
$$

Let us assume that we have the appropriate constraint so that, for general values of $n$, the universal contribution to the Rényi entropies becomes

$$
S_{n}^{\text {univ }}=\log (R / \delta) \int_{\partial V} d^{4} y \sqrt{h}\left[2 f_{A}(n) E_{4}+6 \pi\left(f_{B_{2}}(n)-\frac{f_{B_{1}}(n)}{4}\right) J+f_{B_{3}}(n) T_{3}\right] .
$$

Then with this structure, we find that eq. (3.27) generalizes to

$$
\sigma_{n}^{(6)}=\frac{27 \pi^{3}}{2} f_{B_{3}}(n)
$$

Substituting eq. (3.31) into the above result, we find

$$
\sigma_{n}^{(6)}=\frac{45 \pi^{2}}{512} \frac{h_{n}}{n-1},
$$

in perfect agreement with our conjecture (3.4). Hence our conjecture is satisfied for the class of theories where eq. (3.33) applies. From a different perspective, our conjecture suggests that the naïve generalization of eq. (3.26) to eq. (3.33) for general Rényi entropies is correct for the set of theories satisfying eq. (3.35). That is, at least for these theories, the general structure of $S_{n}^{\text {univ }}$ is given by eq. (3.33) for general regions in six dimensions. 


\section{Discussion}

In this paper, we have generalized our original conjecture $(1.6)[10,12]$, which relates the corner coefficient $\sigma^{(3)}$ with the central charge $C_{\mathrm{T}}$ in the two-point function of the stress tensor. Our generalization provides a similar relation for the coefficient controlling the universal contribution to the EE in almost smooth limit of a (hyper)conical singularity in the entangling surface for CFTs in general dimensions. In particular, we have shown that these cone coefficients $\sigma^{(d)}$ are again determined by $C_{\mathrm{T}}$ through

$$
\sigma^{(d)}=C_{\mathrm{T}} \frac{\pi^{d-1}(d-1)(d-2) \Gamma\left[\frac{d-1}{2}\right]^{2}}{8 \Gamma[d / 2]^{2} \Gamma[d+2]} \times \begin{cases}\pi & d \text { odd }, \\ 1 & d \text { even } .\end{cases}
$$

We were able to prove this relation using the result of [27], which applies for general holographic theories. That is, the proof applies to holographic CFTs that are dual to any higher curvature theory of gravity in the bulk, where the bulk Lagrangian does not contain derivatives of the Riemann tensor. While we have proven this relation for general holographic theories, we conjecture it applies for general CFTs in arbitrary dimensions.

As a consistency check, we have verified that this formula is in agreement with the results obtained using the Ryu-Takayanagi prescription [16-19] for holographic theories dual to Einstein gravity in $d=3,4,6,8,10,12,14$. The odd dimensional cases with $d>3$ are more challenging. In particular, the RT prescription gives rise to very complicated implicit expressions for $a^{(d)}(\Omega)$ for $d=5,7, \cdots$, which have proven impossible to treat analytically as yet. Interestingly, eq. (4.1) provides explicit information on $a^{(d)}(\Omega)$ in these cases. It would be interesting to compute the cone coefficient $\sigma^{(d)}$ for $d=5,7, \cdots$ numerically for these holographic theories and verify that the corresponding values agree with our general formula (4.1) above.

In section 1.1, we built on the above result to extend our conjecture (1.9) for Rényi corner coefficients in $d=3$ [11] to the following expression

$$
\sigma_{n}^{(d)}=\frac{h_{n}}{n-1} \frac{(d-1)(d-2) \pi^{\frac{d-4}{2}} \Gamma\left[\frac{d-1}{2}\right]^{2}}{16 \Gamma[d / 2]^{3}} \times \begin{cases}\pi & d \text { odd }, \\ 1 & d \text { even },\end{cases}
$$

in general dimensions. While we have somewhat less evidence for this result, we again conjecture that it applies for general CFTs. In table 2, we summarize the theories for which this conjecture has been shown to be true so far. These include: for $n=1$, general holographic theories in all dimensions (in the present paper and [21]); for all $n$ for three-dimensional free scalar and fermion fields [10-12, 20, 23, 24]; for all $n$ for all four-dimensional theories satisfying $f_{b}(n)=f_{c}(n)$ (in the present paper and [25]), including e.g., holographic theories and free fields; for all $n$ for all six-dimensional theories where eq. (3.33) holds (in the present paper and $[25,26]$ ), including (at least for $n=1$ ) various holographic theories and e.g., a free $\mathcal{N}=(1,0)$ hypermultiplet. We re-iterate that we have not explicitly established that eq. (4.2) applies for a single free scalar or fermion. It would be interesting to explore the empty slots in table 2 e.g., for five-dimensional and six-dimensional free scalars and fermions. Of course, a more ambitious goal would correspond to finding a complete proof of eq. (4.2) for general CFTs in arbitrary dimensions. 


\begin{tabular}{|c|c|c|c|}
\hline$d$ & Holography & Free Fields & Constrained \\
\hline 3 & $n=1$ & $\forall n$ & N/A \\
\hline 4 & $n=1$ & $\forall n$ & $\forall n$ \\
\hline 5 & $n=1$ & - & N/A \\
\hline 6 & $n=1$ & - & $\forall n$. \\
\hline
\end{tabular}

Table 2. Values of $n$ for which our generalized conjecture eq. (4.2) has been verified so far for $d=3,4,5,6$ theories. The first column corresponds to general holographic theories. The second makes reference to free fields (i.e., a free massless scalar and fermion). The last one corresponds to $d=4$ theories for which $f_{b}(n)=f_{c}(n)$ and $d=6$ theories satisfying $3 f_{B_{3}}(n)=f_{B_{2}}(n)-f_{B_{1}}(n) / 2$ respectively. Here, 'Not Applicable' for $d=3$ and 5 indicates that no analogous constrained theories have been found in these dimensions. Note that in $d=4$, at least certain holographic theories and the free fields fall in the last column but it may well apply for general CFTs. For $d=6$ and $n=1$, at least some holographic theories, but e.g., not the free fields fall in the last column.

Finally, it would be interesting to further explore the implications of our results on the general structure of the universal contributions to Rényi entropy in various dimensions particularly in odd dimensions, for which much less is known in this respect.

\section{Acknowledgments}

We thank to Lorenzo Bianchi, Aitor Lewkowycz, Marco Meineri, Roger Melko, Tomás Ortín, C.S. Shahbazi, Misha Smolkin and William Witczak-Krempa for useful discussions and comments. Research at Perimeter Institute is supported by the Government of Canada through Industry Canada and by the Province of Ontario through the Ministry of Research \& Innovation. The work of PB has been supported by the JAE-predoc grant JAEPre 2011 00452. RCM acknowledges support from an NSERC Discovery grant and funding from the Canadian Institute for Advanced Research.

\section{A Twist operators}

Recall that the twist operator $\tau_{n}$ is defined in the replicated field theory formed as a tensor product of $n$ copies of the original QFT. In particular, it is the codimension-two surface operator extending over the entangling surface, i.e., the boundary of the region $V$, whose expectation value yields

$$
\left\langle\tau_{n}\right\rangle_{n}=\operatorname{Tr}\left[\rho_{V}^{n}\right] .
$$

Here the subscript $n$ on the expectation value on the left-hand side indicates that it is taken in the $n$-fold replicated QFT. Of course, $\tau_{n}$ depends on the region $V$ but we have omitted this dependence here to simplify the notation. For further discussion and details, see e.g., [1, 2, 11, 38, 39].

In the case of a CFT, the conformal scaling dimension $h_{n}$ of the twist operator is defined as the coefficient of the leading power-law divergence in the correlator $\left\langle T_{\mu \nu} \tau_{n}\right\rangle_{n}$ as 
the location of $T_{\mu \nu}$ approaches that of $\tau_{n}[38,39]$. In the case of a twist operator on an infinite (hyper)plane, this correlator is constrained by the residual conformal symmetries and conservation of the stress tensor to take the form:

$$
\begin{aligned}
\left\langle T_{a b} \tau_{n}\right\rangle_{n} & =-\frac{h_{n}}{2 \pi} \frac{\delta_{a b}}{y^{d}}, \quad\left\langle T_{a i} \tau_{n}\right\rangle_{n}=0, \\
\left\langle T_{i j} \tau_{n}\right\rangle_{n} & =\frac{h_{n}}{2 \pi} \frac{(d-1) \delta_{i j}-d \hat{n}_{i} \hat{n}_{j}}{y^{d}}
\end{aligned}
$$

where the indices $a, b$ and $i, j$ denote the $d-2$ parallel directions and the two transverse directions to the twist operator. ${ }^{16}$ Also, $y$ is the perpendicular distance from the stress tensor insertion to the twist operator and $\hat{n}_{i}$ is the unit vector orthogonally directed from $\tau_{n}$ to the stress tensor. Note that $T_{\mu \nu}$ here denotes the stress tensor for the entire $n$-fold replicated CFT.

While the above expressions are only valid for a twist operator on a hyperplane, we stress that in general the leading singularity takes this form whenever $y \ll \ell$, where $\ell$ is any scale entering in the description of the geometry of the entangling surface. Hence the scaling dimension $h_{n}$ is a fixed coefficient which is characteristic of all twist operators $\tau_{n}$ (in a given CFT), independent of the details of the geometry of the corresponding entangling surface. Finally, let us add that $h_{1}=0$ since the twist operator $\tau_{n}$ becomes trivial for $n=1$.

Open Access. This article is distributed under the terms of the Creative Commons Attribution License (CC-BY 4.0), which permits any use, distribution and reproduction in any medium, provided the original author(s) and source are credited.

\section{References}

[1] P. Calabrese and J.L. Cardy, Entanglement entropy and quantum field theory, J. Stat. Mech. (2004) P06002 [hep-th/0405152] [INSPIRE].

[2] P. Calabrese and J.L. Cardy, Entanglement entropy and quantum field theory: A Non-technical introduction, Int. J. Quant. Inf. 4 (2006) 429 [quant-ph/0505193] [INSPIRE].

[3] H. Casini and M. Huerta, Entanglement entropy in free quantum field theory, J. Phys. A 42 (2009) 504007 [arXiv:0905.2562] [INSPIRE].

[4] H. Casini, M. Huerta and L. Leitao, Entanglement entropy for a Dirac fermion in three dimensions: Vertex contribution, Nucl. Phys. B 814 (2009) 594 [arXiv:0811.1968] [INSPIRE].

[5] H. Casini and M. Huerta, Universal terms for the entanglement entropy in $2+1$ dimensions, Nucl. Phys. B 764 (2007) 183 [hep-th/0606256] [INSPIRE].

[6] T. Grover, A.M. Turner and A. Vishwanath, Entanglement Entropy of Gapped Phases and Topological Order in Three dimensions, Phys. Rev. B 84 (2011) 195120 [arXiv:1108.4038] [INSPIRE].

[7] H. Liu and M. Mezei, A Refinement of entanglement entropy and the number of degrees of freedom, JHEP 04 (2013) 162 [arXiv: 1202.2070] [INSPIRE].

\footnotetext{
${ }^{16}$ Let us add that implicitly the above expressions are normalized by dividing by $\left\langle\tau_{n}\right\rangle_{n}$ but we leave this normalization implicit to avoid the clutter that would otherwise be created.
} 
[8] R.C. Myers and A. Singh, Entanglement Entropy for Singular Surfaces, JHEP 09 (2012) 013 [arXiv:1206.5225] [INSPIRE].

[9] H. Casini, M. Huerta, R.C. Myers and A. Yale, Mutual information and the F-theorem, JHEP 10 (2015) 003 [arXiv:1506.06195] [INSPIRE].

[10] P. Bueno, R.C. Myers and W. Witczak-Krempa, Universality of corner entanglement in conformal field theories, Phys. Rev. Lett. 115 (2015) 021602 [arXiv:1505.04804] [INSPIRE].

[11] P. Bueno, R.C. Myers and W. Witczak-Krempa, Universal corner entanglement from twist operators, JHEP 09 (2015) 091 [arXiv: 1507.06997] [INSPIRE].

[12] P. Bueno and R.C. Myers, Corner contributions to holographic entanglement entropy, JHEP 08 (2015) 068 [arXiv: 1505.07842] [INSPIRE].

[13] T. Hirata and T. Takayanagi, AdS/CFT and strong subadditivity of entanglement entropy, JHEP 02 (2007) 042 [hep-th/0608213] [INSPIRE].

[14] E. Fradkin and J.E. Moore, Entanglement entropy of $2 D$ conformal quantum critical points: hearing the shape of a quantum drum, Phys. Rev. Lett. 97 (2006) 050404 [cond-mat/0605683] [INSPIRE].

[15] I.R. Klebanov, T. Nishioka, S.S. Pufu and B.R. Safdi, On Shape Dependence and RG Flow of Entanglement Entropy, JHEP 07 (2012) 001 [arXiv:1204.4160] [INSPIRE].

[16] S. Ryu and T. Takayanagi, Holographic derivation of entanglement entropy from AdS/CFT, Phys. Rev. Lett. 96 (2006) 181602 [hep-th/0603001] [INSPIRE].

[17] S. Ryu and T. Takayanagi, Aspects of Holographic Entanglement Entropy, JHEP 08 (2006) 045 [hep-th/0605073] [INSPIRE].

[18] T. Nishioka, S. Ryu and T. Takayanagi, Holographic Entanglement Entropy: An Overview, J. Phys. A 42 (2009) 504008 [arXiv: 0905.0932] [INSPIRE].

[19] T. Takayanagi, Entanglement Entropy from a Holographic Viewpoint, Class. Quant. Grav. 29 (2012) 153001 [arXiv:1204.2450] [INSPIRE].

[20] H. Elvang and M. Hadjiantonis, Exact results for corner contributions to the entanglement entropy and Rényi entropies of free bosons and fermions in 3d, Phys. Lett. B 749 (2015) 383 [arXiv: 1506.06729] [INSPIRE].

[21] R.-X. Miao, A holographic proof of the universality of corner entanglement for CFTs, JHEP 10 (2015) 038 [arXiv: 1507.06283] [INSPIRE].

[22] M. Alishahiha, A.F. Astaneh, P. Fonda and F. Omidi, Entanglement Entropy for Singular Surfaces in Hyperscaling violating Theories, JHEP 09 (2015) 172 [arXiv:1507.05897] [INSPIRE].

[23] J.S. Dowker, Conformal weights of charged Rényi entropy twist operators for free scalar fields, arXiv: 1508.02949 [INSPIRE].

[24] J.S. Dowker, Note on Rényi vertex contributions and twist operator weights for free scalar fields, arXiv: 1509.00782 [INSPIRE].

[25] A. Lewkowycz and E. Perlmutter, Universality in the geometric dependence of Rényi entropy, JHEP 01 (2015) 080 [arXiv:1407.8171] [INSPIRE].

[26] B.R. Safdi, Exact and Numerical Results on Entanglement Entropy in (5+1)-Dimensional CFT, JHEP 12 (2012) 005 [arXiv: 1206.5025] [INSPIRE]. 
[27] M. Mezei, Entanglement entropy across a deformed sphere, Phys. Rev. D 91 (2015) 045038 [arXiv: 1411.7011] [INSPIRE].

[28] A. Allais and M. Mezei, Some results on the shape dependence of entanglement and Rényi entropies, Phys. Rev. D 91 (2015) 046002 [arXiv:1407.7249] [INSPIRE].

[29] R.C. Myers and A. Sinha, Seeing a c-theorem with holography, Phys. Rev. D 82 (2010) 046006 [arXiv: 1006.1263] [INSPIRE].

[30] R.C. Myers and A. Sinha, Holographic c-theorems in arbitrary dimensions, JHEP 01 (2011) 125 [arXiv: 1011.5819] [INSPIRE].

[31] H. Casini, M. Huerta and R.C. Myers, Towards a derivation of holographic entanglement entropy, JHEP 05 (2011) 036 [arXiv:1102.0440] [INSPIRE].

[32] J.L. Cardy, Is there a c-theorem in four dimensions?, Phys. Lett. B 215 (1988) 749 [INSPIRE].

[33] I.R. Klebanov, S.S. Pufu and B.R. Safdi, F-Theorem without Supersymmetry, JHEP 10 (2011) 038 [arXiv: 1105.4598] [INSPIRE].

[34] D.L. Jafferis, I.R. Klebanov, S.S. Pufu and B.R. Safdi, Towards the F-Theorem: $N=2$ Field Theories on the Three-Sphere, JHEP 06 (2011) 102 [arXiv:1103.1181] [INSPIRE].

[35] H. Osborn and A.C. Petkou, Implications of conformal invariance in field theories for general dimensions, Annals Phys. 231 (1994) 311 [hep-th/9307010] [INSPIRE].

[36] X. Dong, Holographic Entanglement Entropy for General Higher Derivative Gravity, JHEP 01 (2014) 044 [arXiv: 1310.5713] [INSPIRE].

[37] J. Camps, Generalized entropy and higher derivative Gravity, JHEP 03 (2014) 070 [arXiv:1310.6659] [INSPIRE].

[38] L.-Y. Hung, R.C. Myers, M. Smolkin and A. Yale, Holographic Calculations of Rényi Entropy, JHEP 12 (2011) 047 [arXiv:1110.1084] [INSPIRE].

[39] L.-Y. Hung, R.C. Myers and M. Smolkin, Twist operators in higher dimensions, JHEP 10 (2014) 178 [arXiv: 1407.6429] [INSPIRE].

[40] E. Perlmutter, A universal feature of CFT Rényi entropy, JHEP 03 (2014) 117 [arXiv:1308.1083] [INSPIRE].

[41] J. Lee, L. McGough and B.R. Safdi, Rényi entropy and geometry, Phys. Rev. D 89 (2014) 125016 [arXiv: 1403.1580 ] [INSPIRE].

[42] S.N. Solodukhin, Entanglement entropy, conformal invariance and extrinsic geometry, Phys. Lett. B 665 (2008) 305 [arXiv: 0802.3117] [INSPIRE].

[43] I.R. Klebanov, S.S. Pufu, S. Sachdev and B.R. Safdi, Rényi Entropies for Free Field Theories, JHEP 04 (2012) 074 [arXiv:1111.6290] [INSPIRE].

[44] D.V. Fursaev, Entanglement Rényi Entropies in Conformal Field Theories and Holography, JHEP 05 (2012) 080 [arXiv: 1201.1702] [INSPIRE].

[45] H. Casini and M. Huerta, Entanglement entropy for the n-sphere, Phys. Lett. B 694 (2010) 167 [arXiv: 1007.1813] [inSPIRE].

[46] D.V. Fursaev and G. Miele, Finite temperature scalar field theory in static de Sitter space, Phys. Rev. D 49 (1994) 987 [hep-th/9302078] [INSPIRE]. 
[47] L. De Nardo, D.V. Fursaev and G. Miele, Heat kernel coefficients and spectra of the vector Laplacians on spherical domains with conical singularities, Class. Quant. Grav. 14 (1997) 1059 [hep-th/9610011] [INSPIRE].

[48] M. Henningson and K. Skenderis, The Holographic Weyl anomaly, JHEP 07 (1998) 023 [hep-th/9806087] [INSPIRE].

[49] M. Henningson and K. Skenderis, Holography and the Weyl anomaly, Fortsch. Phys. 48 (2000) 125 [hep-th/9812032] [INSPIRE].

[50] F. Bastianelli, S. Frolov and A.A. Tseytlin, Conformal anomaly of $(2,0)$ tensor multiplet in six-dimensions and AdS/CFT correspondence, JHEP 02 (2000) 013 [hep-th/0001041] [INSPIRE].

[51] L.-Y. Hung, R.C. Myers and M. Smolkin, On Holographic Entanglement Entropy and Higher Curvature Gravity, JHEP 04 (2011) 025 [arXiv:1101.5813] [INSPIRE]. 